\title{
Association of Thyroid Function with Body Mass Index in Adolescent Girls
}

\author{
Ritu Priya ${ }^{1}$, Suprava Patel ${ }^{2}$, Shashi S Dubey ${ }^{3}$, Rachita Nanda ${ }^{4}$, Eli Mohapatra ${ }^{5}$
}

\begin{abstract}
Introduction: Body mass index (BMI) is an important tool for monitoring development in the growing age group. Thyroid hormone affects body weight through changes in basal metabolic rate (BMR). The increasing prevalence of deranged BMI in young girls' necessities to rule out thyroid dysfunction.

Aim: The study objective was to analyze the association between BMI and thyroid function in adolescent girls.

Materials and methods: Seventy-five girls of 12-18 years of age group were included in this study. All participants had undergone anthropometric measurements and serum analysis for thyroid-stimulating hormone (TSH) and T4. Based on the growth and BMI chart of the Indian Association of Pediatrics (IAP), the participants were categorized into two groups. Participants with BMI within 25-75 percentiles were categorized as group I and those below 25 and above 75 percentile were categorized as group II.

Results: The median values of age, weight, and TSH levels were significantly higher in group II participants when compared with group I. In group II, serum TSH and BMI depicted a highly significant positive correlation ( $p$ value $=0.001$ ) while in group I this correlation was 0.044 .

Conclusion: Serum TSH was found elevated in participants with BMI derangement. The stronger correlation between TSH and BMI suggested regarding the inter-relationship between thyroid hormone and BMI and impact of each other's metabolism.

Clinical significance: The subclinical serum TSH levels and its correlation with BMI require more detailed study to devise a causal relationship. Thyroid hormones have a role in the development and any derangement in the adolescents affects the development.

Keywords: Body mass index, Correlation, Cross-sectional study, Thyroid-stimulating hormone.

Indian Journal of Medical Biochemistry (2020): 10.5005/jp-journals-10054-0158
\end{abstract}

\section{INTRODUCTION}

The effect of thyroid hormones on body mass index (BMI) has been studied by various researchers, and it has been demonstrated that overt thyroid dysfunction affects body weight. ${ }^{1-3}$ The increasing prevalence of obesity in the population particularly in younger individuals can affect the metabolism and may lead to many conditions like diabetes, cardiovascular risk, etc. Although the effects of hypothyroidism and hyperthyroidism on body weight have been seen in various studies, the cause-effect relationship has not yet been explained. The adolescent period is an important stage of development. Thyroid hormones have a major role during this stage. Thyroid hormones are essential for normal growth, sexual development, and reproductive function. During puberty, changes in thyroid functions and an increase in thyroid volume occur as an adaptation to physical development and reproductive maturation. Any small derangement in thyroid hormone profile during this period will affect the individual's overall growth, especially BMI. ${ }^{4,5}$ Therefore, this study was aimed to investigate the change in serum thyroid-stimulating hormone (TSH) and T4 levels with respect to $\mathrm{BMI}$ in adolescent girls.

\section{Materials and Methods}

It was a cross-sectional study done at the Department of Biochemistry, All India Institute of Medical Sciences (AIIMS), Raipur. Seventy-five girls of 12-18 years who had visited the hospital OPD accompanying along with the patients were included in this study. The study duration was for a period of 6 months.

Girls with any medical history of disease related to thyroid, cardiovascular risk, hypertension, diabetes, any chronic illness,

\author{
1,2,4,5 Department of Biochemistry, All India Institute of Medical \\ Sciences, Raipur, Chhattisgarh, India \\ ${ }^{3}$ MBBS, All India Institute of Medical Sciences, Raipur, Chhattisgarh, \\ India
}

Corresponding Author: Suprava Patel, Department of Biochemistry, All India Institute of Medical Sciences, Raipur, Chhattisgarh, India, e-mail:dr_suprava@yahoo.co.in

How to cite this article: Priya R, Patel S, Dubey SS, et al. Association of Thyroid Function with Body Mass Index in Adolescent Girls. Indian J Med Biochem 2020;24(2):55-58.

Source of support: Nil

Conflict of interest: None

any endocrinopathy, and family history of thyroid disorder were excluded from the study. The study was approved by the Institutional Ethics Committee. Written informed consent from the parents/guardians/legal representatives of all participants was taken for enrollment into the study.

All participants included in this study were subjected to a questionnaire for detailed history, complete clinical examination, and laboratory investigations.

- Height:The height of an individual, participant of the study was measured, by a stadiometer without shoes.

- Weight: The weight of participants was measured by a digital weighing machine.

- BMI: Body mass index was calculated by the formula BMI= weight in $\mathrm{kg} / \mathrm{height}^{2}$ in meter. Body mass index in the range of 25 to 75 percentiles according to the Indian Association of 
Paediatrics (IAP) was defined as normal BMI. Those participants with calculated BMI was within this range were categorized as group I. Individuals below 25 and above 75 percentile were referred to as abnormal BMI and categorized as group II.

- Thyroid function test: Thyroid-stimulating hormone and total T4 were estimated by Cobas e411 based on electrochemiluminescence assay (ECLIA). The biological reference range for serum TSH and T4, according to kit inserts, was considered to be $0.51-4.3(\mu \mathrm{lU} / \mathrm{mL})$ and $0.76-1.7(\mathrm{ng} / \mathrm{dL})$, respectively.

Results were statistically analyzed by SPSS 20.0 for Windows. All the variables were presented as median with interquartile range (25th percentile to 75 th percentile) due to the non-Gaussian distribution of variables. The Kolmogorov-Smirnov method was used to test for normality of data distribution. Mann-Whitney $U$ test was used to compare the results of the studied groups.

The associations between the variables in a group were analyzed using the Spearman test as a correlation coefficient $(r)$ and their significance ( $p$ value). Results were considered significant when the $p$ value was $<0.05$.

\section{Results}

The percentage of individuals with abnormal BMI was 42.67\%, which included underweight, overweight and obese girls (Fig. 1). The median values with an interquartile range of all studied parameters, in all studied groups, are shown in Table 1.

As depicted in Table 2, the age difference was significant between the groups. The table reflected a significant difference in weight and BMI between the two groups. The median value of TSH is increased with altered BMI.

The box size for TSH between the two groups is dissimilar but the median value almost the same as reflected in Figure $2 \mathrm{~A}$. The lower quartile is smaller than the upper quartile showing the distribution of TSH more toward the subclinical range in group II. The size of the box is the same in both groups with an almost equal median for T4 between the two groups (Fig. 2B).

The regression fit line in the scatter plot for TSH and BMI of group II subjects decoded a strong positive correlation with serum TSH ( $p=0.001)$ when compared with group I subjects ( $p=0.04)$ as shown in Table 2, Figs 1 and 2. As depicted in Figures 5 and 6 , serum total T4 depicted an insignificant but negative correlation with $\mathrm{BMI}$ in group II subjects.

\section{Discussion}

The percentage of adolescent girls in the study with deranged BMI was $42.67 \%$ (Fig. 1), which included underweight, overweight, and obese girls. This finding is similar to the presence of abnormal weight in $49.31 \%$ in the study by Fox et al. ${ }^{6}$ The obesity prevalence in India as per the study conducted by the School of Public Health, London was published to be nearly $16-20 \%{ }^{7}$ This difference could be due to the difference in BMI criteria taken into consideration for the study.

A significant increase in weight in comparison to height, as depicted in Table 2, implied that changes in BMI could be due to the change in weight as age increases. The skeletal weight, muscle mass, and adipose tissue are a prime contributor to body composition, and changes in these frequently regulate body weight. ${ }^{8}$ Among these, fat accumulation has an important role because girls in the age group are under influence of hormone interplay that triggers
Table 1: The demographic data and laboratory parameters of all participants in the study groups

\begin{tabular}{ll}
\hline Parameters & Median (interquartile range) \\
\hline Age (years) & $15(13-17)$ \\
Height $(\mathrm{cm})$ & $158.5(151.8-167.2)$ \\
Weight $(\mathrm{kg})$ & $49(42-62)$ \\
$\mathrm{BMI}\left(\mathrm{kg} / \mathrm{m}^{2}\right)$ & $20(18-23)$ \\
$\mathrm{TSH}(\mu \mathrm{lU} / \mathrm{mL})$ & $2.60(1.84-3.6)$ \\
Total T4 $(\mathrm{ng} / \mathrm{dL})$ & $10.1(7.9-11.5)$ \\
\hline
\end{tabular}

Table 2: Comparison of the demographic data and laboratory parameters between the study groups

\begin{tabular}{llll}
\hline Parameters & Group I $(n=43)$ & Group II $(n=32)$ & p value \\
\hline Age (years) & $14(13-17)$ & $16(15-18)$ & $0.022^{*}$ \\
Height $(\mathrm{cm})$ & $157(152-162)$ & $162(152-170)$ & 0.164 \\
Weight $(\mathrm{kg})$ & $47(41-50)$ & $64(51-70)$ & $<0.001^{* * *}$ \\
$\mathrm{BMI}\left(\mathrm{kg} / \mathrm{m}^{2}\right)$ & $18(17-20)$ & $23.5(22-25)$ & $<0.001^{* * *}$ \\
TSH $(\mu \mathrm{lU} / \mathrm{mL})$ & $2.3(1.7-3.2)$ & $3.6(2.2-7.3)$ & $0.005^{* *}$ \\
Total T4 $(\mathrm{ng} / \mathrm{dL})$ & $10.4(7.9-11.6)$ & $9.7(7.8-11.5)$ & 0.447 \\
\hline
\end{tabular}

$*{ }^{* * * * * *}$ Shows strength of significance

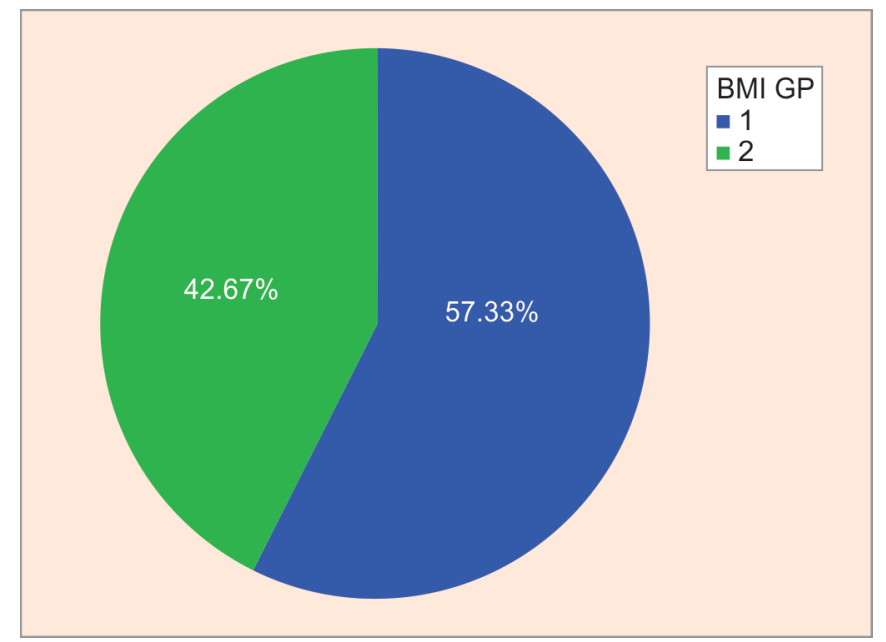

Fig. 1: Scatter plot showing correlation between TSH and BMI in group I $\left(r=0.31, p=0.04^{*}\right)$

physical alterations like an accumulation of fat deposits around the hip and breasts required for reproductive maturation. ${ }^{9}$

The statistically significant difference between age, weight, and TSH levels between the two groups indicated that with growing age, thyroid hormones have a regulatory impact on fat metabolism. The strong positive correlation between serum TSH and BMI and insignificant but negative correlation of serum T4 with BMI in group II subjects suggested that any sort of alteration in thyroid hormones may cause an increase in body weight. Our observation was in accordance with other studies. ${ }^{5,10-12}$ Serum TSH is being used as a primary screening parameter in the clinical setting to diagnose thyroid-related disorders at the subclinical stage. In the present study, serum TSH value was significantly different between the groups, and the distribution of TSH in group II individuals was found to be more in the subclinical range (Fig. 2A). Nearly $16 \%$ of girls in group II had serum TSH in the subclinical range. This signifies that most of the teenage girls with altered BMI had compromised 

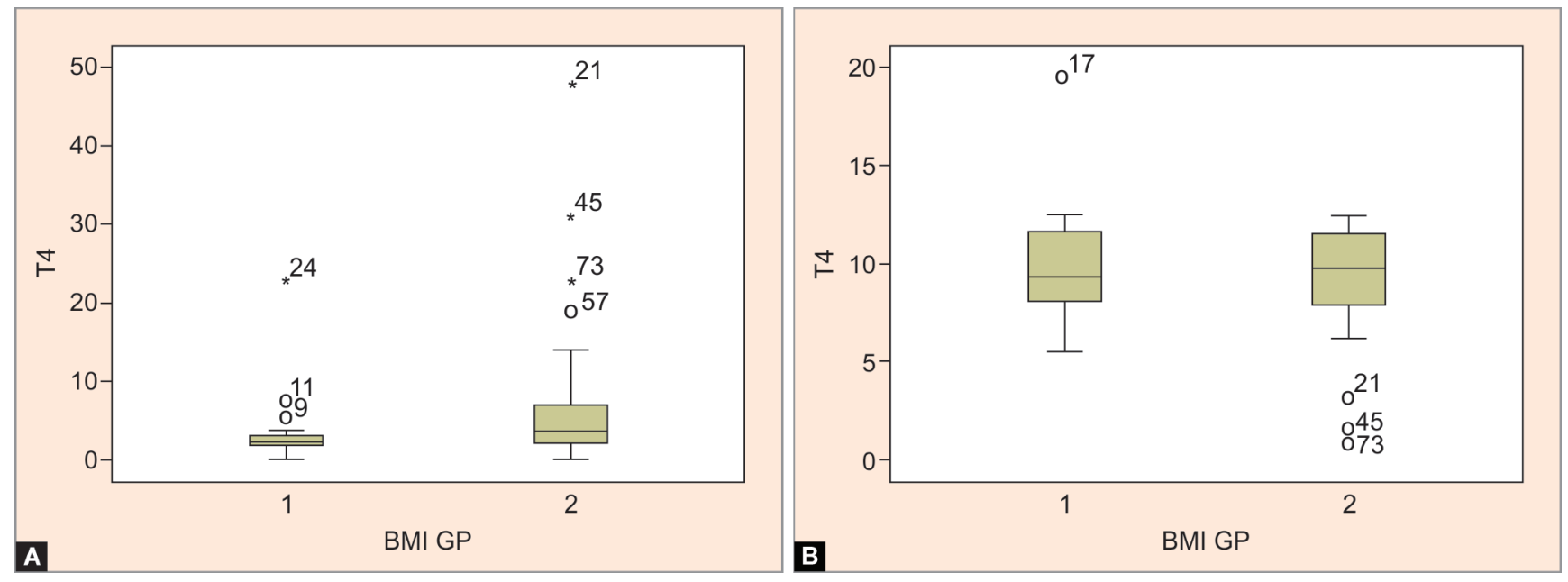

Fig. 2: Box plot with distribution of TSH and T4 between the groups showing median with interquartile range. Star represents the outliers

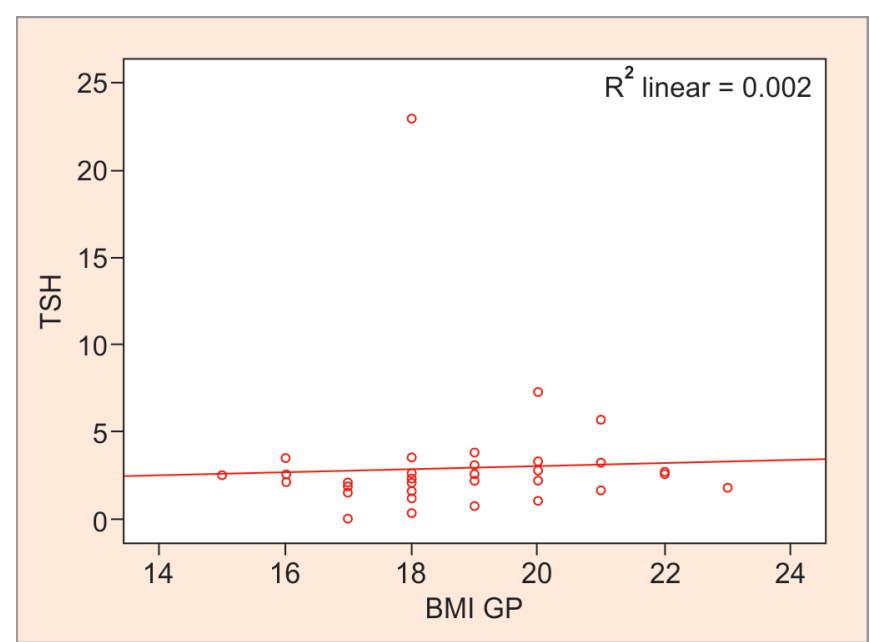

Fig. 3: Scatter plot showing correlation between TSH and BMI in group I $\left(r=0.31, p=0.04^{*}\right)$

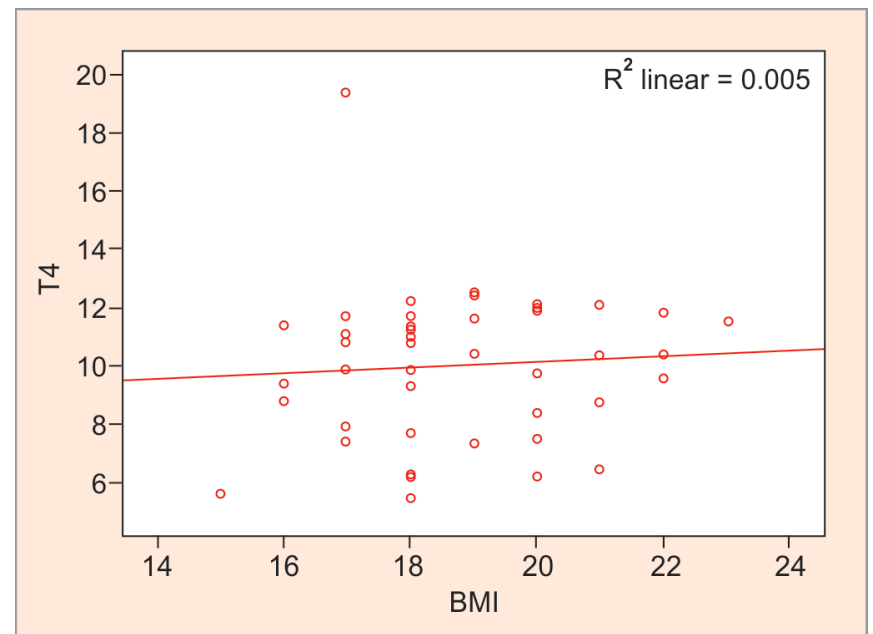

Fig. 5: Scatter plot showing correlation between total T4 and BMI in group I $(r=0.131, p=0.403)$

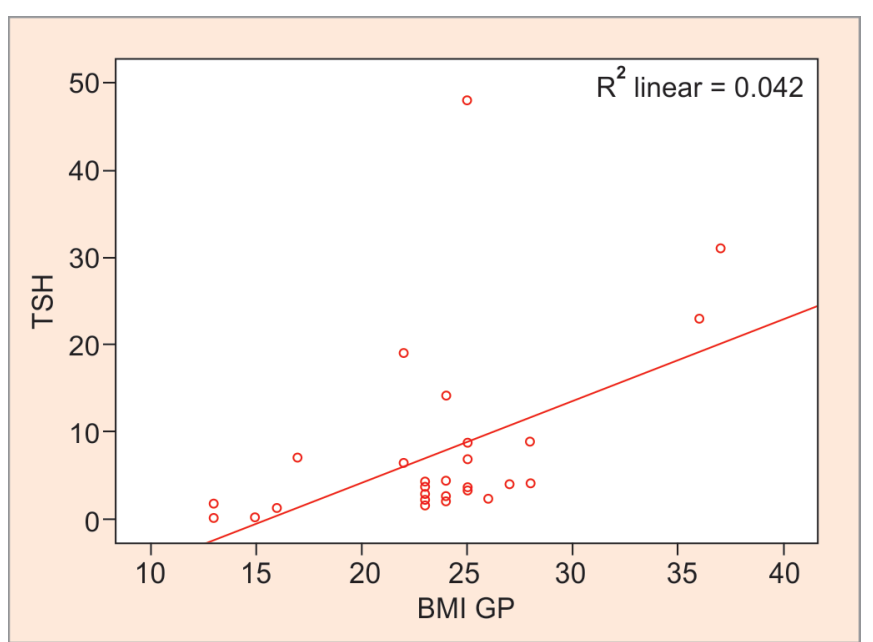

Fig. 4: Scatter plot showing correlation between TSH and BMI in group II $\left(r=0.565, p=0.001^{* * *}\right)$

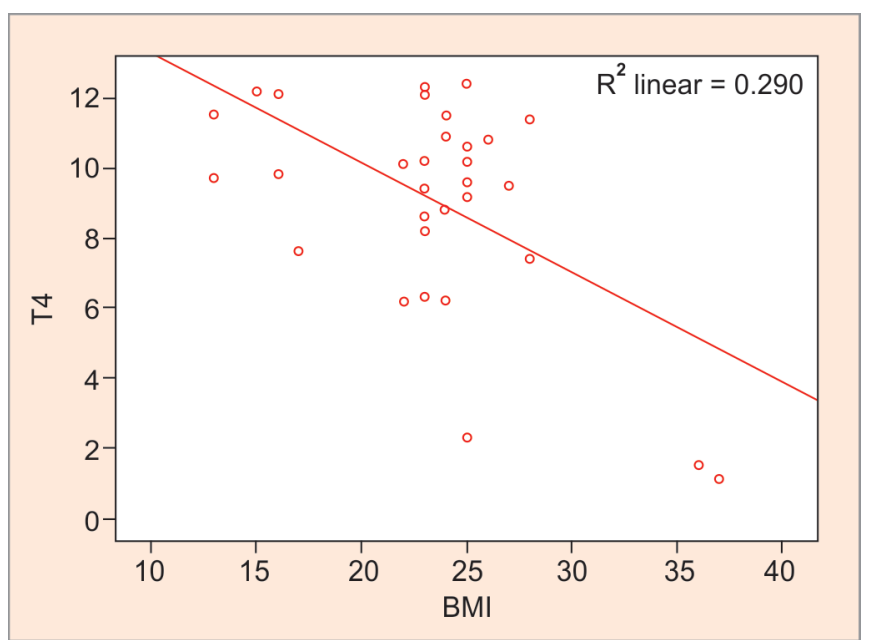

Fig. 6: Scatter plot showing correlation between total T4 and BMI in group II $(r=-0.277, p=0.12)$ 
thyroid status (Fig. 2). Studies have been published with data that even unit change in TSH level can alter the components of fat metabolism by $3 \% .^{13,14}$

Thyroid hormones have an association with weight and adipose tissue. Hypothyroidism is associated with a decrease in metabolic rate and affects both lipid and carbohydrate metabolism. ${ }^{15}$ Adipose tissues express receptors for thyroid hormones. Thyroidstimulating hormone receptors are expressed during lineagespecific differentiation of preadipocyte precursors into mature fat cells. Studies have also explained regarding TSH receptor expression during adipogenesis but decrease with completion of differentiation and with fat accumulation. ${ }^{5}$ Reduced expression of receptors induces peripheral tissue resistance and interferes with the activity of TSH and thyroid hormones on adipocytes leading to a compensatory increase in TSH and total T4. ${ }^{16}$ Excessive fat deposition and obesity induce deiodinase activity for the peripheral conversion of T4 to T3 and increase energy expenditure as a defense mechanism to counteract fat accumulation. ${ }^{5,16}$ Various studies have put forth the theory of elevated leptin in obese individuals, which stimulates TRH secretion and eventually TSH secretion. ${ }^{10,17}$ Thyroid hormones are involved in both lipogenesis and lipolysis, an effect that possibly is mediated by affecting local noradrenaline levels and/or adrenergic post-receptor signaling. ${ }^{11}$ Zhang et al. described that the risk of obesity is quite higher in patients with subclinical hypothyroidism in Chinese adolescents. ${ }^{18}$ Solanki et al. reported that the level of TSH is quite higher in obese patients and it increases as BMI increases as seen in this study. ${ }^{5}$ Velivala et al. found a higher prevalence of $\mathrm{SCH}$ is higher in females and increased with $\mathrm{BMI} .{ }^{19}$

\section{Conclusion}

The variations of normal thyroid function with differences in BMI may be due to the changes in resting energy consumption. The positive correlation between TSH and BMI indicates the regulatory role of thyroid hormones on weight maintenance and vice versa. Gradual weight gain in an individual should not be overlooked rather thyroid dysfunction needs to be ruled out.

\section{Limitation of Study}

The limitation of the study includes a small sample size and incomplete thyroid profile. Further large-scale studies are required to establish a causal relationship between obesity and thyroid hormones.

\section{References}

1. Ghergherehchi R, Hazhir N. Thyroid hormonal status among children with obesity. Ther Adv Endocrinol Metab 2015;6(2):51-55. DOI: 10.1177/2042018815571892.

2. Longhi S, Radetti G. Thyroid function and obesity. J Clin Res Pediatr Endocrinol 2013;5(Suppl 1):40-44. DOI: 10.4274/jcrpe.856.

3. Namburi R, Ponnala A, Palanki R, et al. Association of obesity and thyrotropinemia in children and adolescents. Thyroid Res Pract 2014;11(2):45-48. DOI: 10.4103/0973-0354.129649.
4. An YM, Moon SJ, Kim SK, et al. Thyroid function in obese Korean children and adolescents: Korea National Health and Nutrition Examination Survey 2013-2015. Ann Pediatr Endocrinol Metab 2018;23(3):141-147. DOI: 10.6065/apem.2018.23.3.141.

5. Solanki A, Bansal S, Jindal S, et al. Relationship of serum thyroid stimulating hormone with body mass index in healthy adults. Indian J Endocrinol Metab 2013;17(Suppl 1):S167-S169. DOI: 10.4103/22308210.119560.

6. Fox CS, Pencina MJ, D'Agostino RB, et al. Relations of thyroid function to body weight: cross-sectional and longitudinal observations in a community-based sample. Arch Intern Med 2008;168(6):587-592. DOI: 10.1001/archinte.168.6.587.

7. Abarca-Gómez L, Abdeen ZA, Hamid ZA, et al. Worldwide trends in body-mass index, underweight, overweight, and obesity from 1975 to 2016: a pooled analysis of 2416 population-based measurement studies in 128.9 million children, adolescents, and adults. Lancet 2017;390(10113):2627-2642. DOI: 10.1016/S0140-6736(17)32129-3.

8. Bergh $\mathrm{IH}$, Skare $\varnothing$, Aase $A$, et al. Weight development from age 13 to 30 years and adolescent socioeconomic status: The Norwegian longitudinal health behaviour study. Int J Public Health 2016;61:465473. DOI: 10.1007/s00038-015-0748-x.

9. Todd AS, Street SJ, Ziviani J, et al. Overweight and obese adolescent girls: the importance of promoting sensible eating and activity behaviors from the start of the adolescent period. Int J Environ Res Public Health 2015;12(2):2306-2329. DOI: 10.3390/ijerph120202306.

10. Bjergved L, Jørgensen $T$, Perrild $H$, et al. Thyroid function and body weight: a community-based longitudinal study. PLoS One 2014;9(4):e93515. DOI: 10.1371/journal.pone.0093515.

11. Knudsen N, Laurberg P, Rasmussen LB, et al. Small differences in thyroid function may be important for body mass index and the occurrence of obesity in the population. J Clin Endocrinol Metab 2005;90(7):4019-4024. DOI: 10.1210/jc.2004-2225.

12. Svare A, Nilsen TIL, Bjøro T, et al. Serum TSH related to measures of body mass: longitudinal data from the HUNT study, Norway. Clin Endocrinol (Oxf) 2011;74(6):769-775. DOI: 10.1111/j.13652265.2011.04009.x.

13. Waring AC, Rodondi N, Harrison S, et al. Thyroid function and prevalent and incident metabolic syndrome in older adults: the health, ageing and body composition study. Clin Endocrinol (Oxf) 2012;76(6):911-918. DOI: 10.1111/j.1365-2265.2011.04328.x.

14. Sami A, Iftekhar MF, Rauf MA, et al. Subclinical hypothyroidism among local adult obese population. Pak J Med Sci 2018;34(4):980-983. DOI: 10.12669/pjms.344.14127.

15. Biondi B. Thyroid and obesity: an intriguing relationship. J Clin Endocrinol Metab 2010;95(8):3614-3617. DOI: 10.1210/jc.20101245.

16. Cicatiello AG, Di Girolamo D, Dentice M. Metabolic effects of the intracellular regulation of thyroid hormone: old players, new concepts. Front Endocrinol 2018;9:474. DOI: 10.3389/fendo.2018.00474.

17. Ambrosi $B$, Masserini $B$, lorio $L$, et al. Relationship of thyroid function with body mass index and insulin-resistance in euthyroid obese subjects. J Endocrinol Invest 2010;33(9):640-643. DOI: 10.1007/ BF03346663.

18. Zhang J, Jiang R, Li L, et al. Serum thyrotropin is positively correlated with the metabolic syndrome components of obesity and dyslipidemia in Chinese adolescents. Int J Endocrinol 2014; 2014:289503. DOI: 10.1155/2014/289503.

19. Velivala AP, Plagudu L, Koyyala VP. Subclinical hypothyroidism in obese patients in rural general hospital. IOSR J Dent Med Sci 2013;5(4):8-10. DOI: 10.9790/0853-0540810. 